\title{
Split ring resonator based THz-driven electron streak camera featuring femtosecond resolution Justyna Fabiańska $^{* a}$, Günther Kassier ${ }^{\mathrm{b}}$, Thomas Feurer ${ }^{\mathrm{a}}$ \\ ${ }^{a}$ Institute of Applied Physics, University of Bern, 3012 Bern, Switzerland \\ ${ }^{\mathrm{b}}$ Max Planck Institute for the Structure and Dynamics of Matter, Bldg. 99, Luruper Chausee 149, 22761 Hamburg, Germany
}

\begin{abstract}
Through combined three-dimensional electromagnetic and particle tracking simulations we demonstrate a THz driven electron streak camera featuring a temporal resolution down to a femtosecond. The ultrafast streaking field is generated in a resonant THz sub-wavelength antenna, which is illuminated by an intense single-cycle $\mathrm{THz}$ pulse. Since electron bunches and $\mathrm{THz}$ pulses are generated by the same laser system, synchronization between the two is inherently guaranteed.
\end{abstract}

Keywords: electron bunch, fs temporal resolution, metamaterials, terahertz optics, ultrafast photonics

\section{INTRODUCTION}

One of the most challenging aims in nature is to watch atomic motion in real time, for example to directly observe chemical processes. This requires sub-picosecond time resolution as well as sufficient spatial resolution ${ }^{1}$. A promising methodology to achieve this goal is ultrafast electron diffraction (UED), which has recently been reviewed by Miller, ${ }^{1,2}$. Valuable work in this field includes studies on chemical reactions in the gas phase ${ }^{3}$, ultrafast melting dynamics of metals $^{4,5}$, ultrafast dynamics in charge density wave materials ${ }^{6,7}$, and insulator to metal phase transitions in organic salts ${ }^{8}$. The success of these experiments has depended heavily on advances in ultrafast electron source technology. Ultrafast generation of electrons and ultrafast manipulation of their trajectories is a rapidly developing field and is tightly intertwined with ultrafast laser technology. Ultrafast lasers illuminate metallic photocathodes or nanotips and produce ultrashort electron bunches. In the 10-100 kV electron energy regime, pulsed electron guns based on planar photocathodes are capable of producing electron bunches with pulse durations in the few hundred femtosecond regime $\mathrm{e}^{4}$, 9-12 while nanotip sources ${ }^{13-17}$ and bunch recompression based systems ${ }^{8,18-20}$ are capable of reaching the 10-100 fs time domain. Several publications report on both conceptual and experimental realizations of temporal electron pulse characterisation in the femtosecond regime. Streak cameras have been applied initially in single-shot mode, to obtain sub-picosecond pulse duration measurements ${ }^{5,10}$. Subsequently, Kassier et al. demonstrated a low-jitter streak camera suitable for accumulation mode measurements with a temporal resolution of $150 \mathrm{fs}^{12}$.

Here, we propose a route to accomplish temporal electron bunch characterization with the potential capability of achieving few-femtosecond temporal resolution for electron energies in the $10-100 \mathrm{kV}$ range as well as relativistic electrons in the $\mathrm{MeV}$ range ${ }^{21}$. We combine resonant $\mathrm{THz}$ sub-wavelength structures, i.e. split-ring resonators, with intense single-cycle $\mathrm{THz}$ pulses. Since electron bunches and $\mathrm{THz}$ pulses are generated with the same laser system, synchronization between the two is inherently guaranteed. The THz split-ring resonator is designed to feature a resonant response and a field enhancement in order to alleviate the demands on the driving laser. The resonance frequencies can be easily tuned throughout the entire $\mathrm{THz}$ spectrum by changing the structure's geometry ${ }^{21,22}$. 


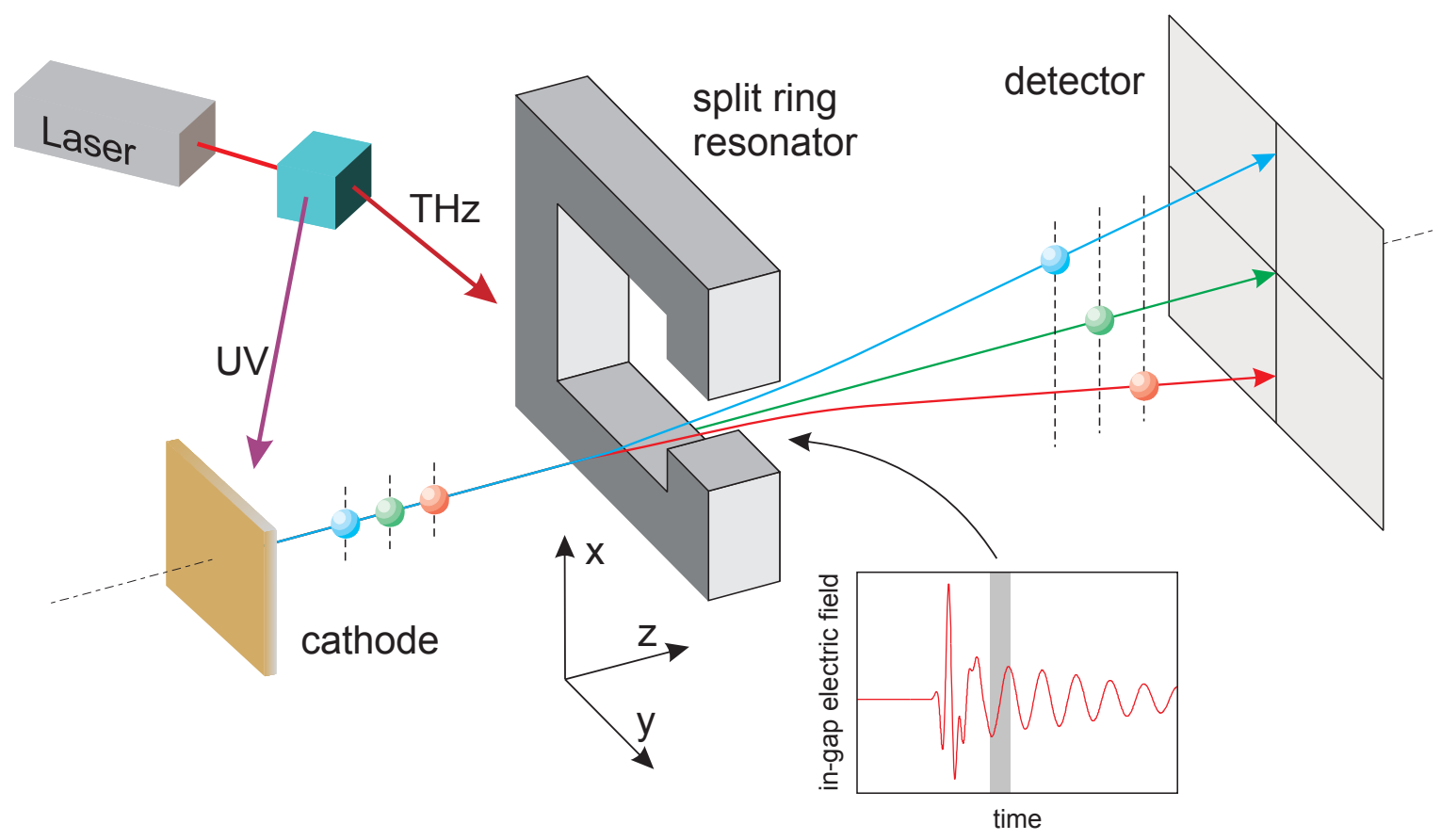

Figure 1. Schematic of the experimental setup.

Figure 1 shows a schematic of the $\mathrm{THz}$ streak camera. While part of the driving laser produces the electron bunches, the other part generates intense single-cycle $\mathrm{THz}$ pulses which are subsequently focused to the split-ring resonator. Field strengths on the order of tens of $\mathrm{MV} / \mathrm{m}$ have been reported at diffraction-limited $\mathrm{THz}$ focal spots $^{23,24}$. If the split-ring resonator is positioned at the focal spot, the resonantly absorbed $\mathrm{THz}$ radiation induces a current flow in the ring that results in an accumulation of charge carriers across the gap region. A simulated electric field distribution is shown in Figure 2. The in-gap field distribution is very close to that of a parallel-plate capacitor. Effectively, the split ring serves as an efficient resonant antenna absorbing the incident $\mathrm{THz}$ radiation and concentrating it in the gap volume thereby increasing the field strength. For a gap area of $10 \mu \mathrm{m}$ by $10 \mu \mathrm{m}$ and a resonance frequency of $0.3 \mathrm{THz}$ the field enhancement is approximately 100 , boosting the maximum attainable electric fields of today's $\mathrm{THz}$ sources to the GV/m $\operatorname{level}^{22,25}$. Since no $\mathrm{THz}$ detection system has the spatial resolution required to directly measure, let alone map out, the electric field distribution in the gap volume, we resort to quantify the charge distribution in the ring, which is responsible for the capacitive charging of the gap, and we extract the in-gap electric field and field enhancement ${ }^{21,22}$. 

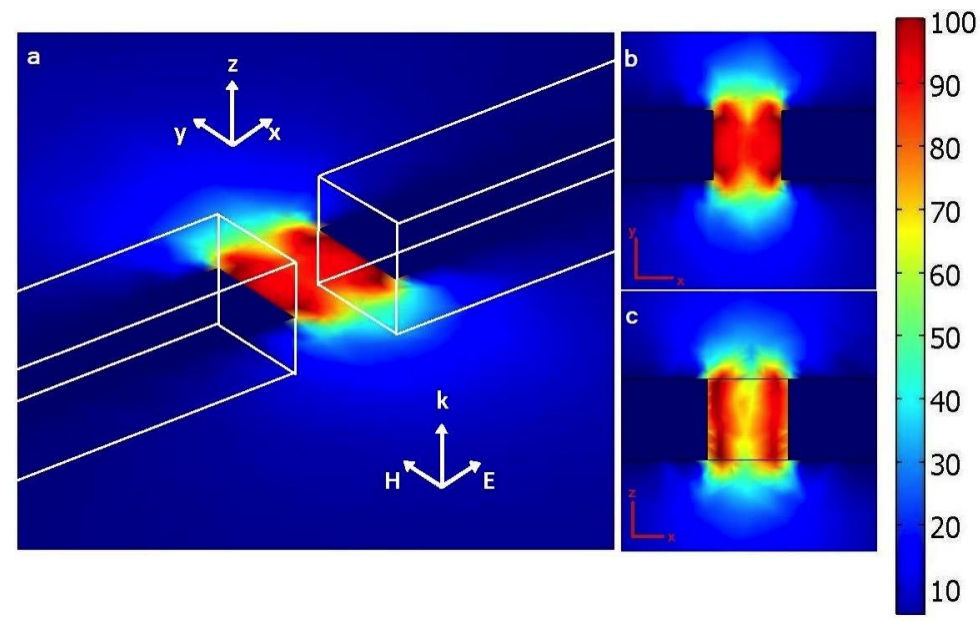

Figure 2. Simulated electric field distribution. (a) $\mathrm{E}_{\mathrm{x}}$ component normalized to the incident field in a plane through the middle of the gap of the split-ring resonator (SRR) at $300 \mathrm{GHz},(b)$ horizontal and (c) vertical field distributions across the gap.

The electron pulses pass through this gap region approximately at the zero-crossing of the in-gap electric field and are deflected by the time varying electric field distribution ${ }^{21}$. Finally, the electrons are detected by a spatially resolving detector, e.g. a micro channel plate (MCP), with the x-axis now being directly proportional to the time axis.

\section{RESULTS}

We analyse the performance of the THz-driven streak camera with respect to angular streak velocity, time resolution, and intrinsic time smearing due to, for example, electric field inhomogeneities. Initially, we assume the actual electron bunches to consist of 1000 quasi-randomly distributed electrons with a square transverse distribution of $4 \mu \mathrm{m} \times 4 \mu \mathrm{m}$ and a zero transverse emittance. We first neglect space charge forces and fix the electron energy to $30 \mathrm{keV}$ and the temporal duration of the initial bunch length in the $0-100 \mathrm{fs}$ range. After passing through the split-ring's gap the electron bunches propagate for $20 \mathrm{~mm}$ before their transverse spatial distribution is measured. The parameters of importance such as angular streak velocity and temporal resolution are invariant with respect to the chosen detector distance but we assume more realistic camera lengths in the subsequent section with simulations that take account of space charge ${ }^{21}$.

Figure 3 depicts the simulated transverse electron distributions for a peak electric field of $100 \mathrm{MV} / \mathrm{m}$. Subfigures a) and b) correspond to the $f_{r}=0.3 \mathrm{THz}$ split ring resonance frequency and electron pulse durations of $0 \mathrm{fs}$ and $50 \mathrm{fs}$, respectively. Similarly, subfigures c) and d) represent the $f_{r}=0.6 \mathrm{THz}$ case and e) and f) the $f_{r}=0.9 \mathrm{THz}$ case. Showing for comparison the 0 -fs pulse duration case allows one to distinguish between effects of the streak field and distortion effects due to transverse beam size. From figure 3 it is already apparent that at a fixed peak electric field, here 100 $\mathrm{MV} / \mathrm{m}$, the streak velocity increases with the resonance frequency. 
a)

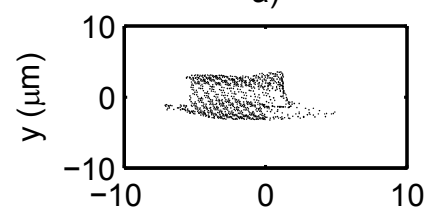

c)

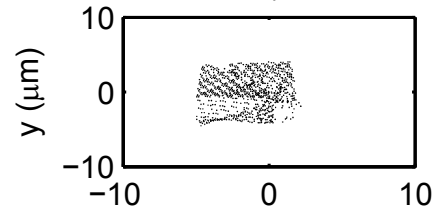

e)

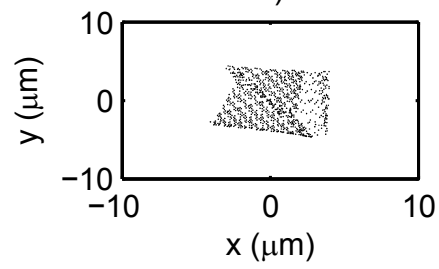

b)

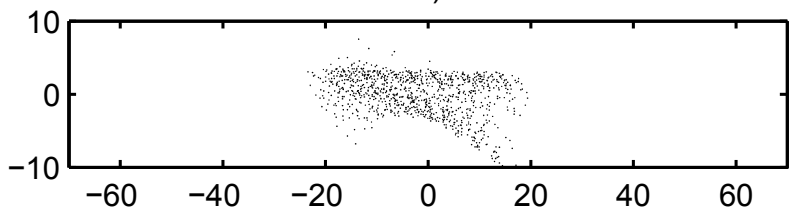

d)

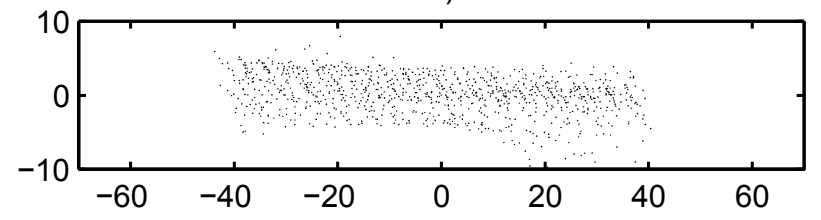

f)

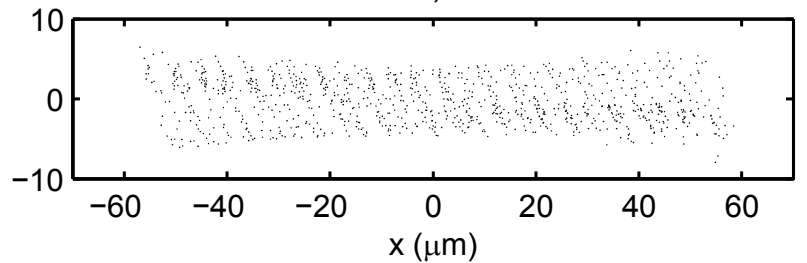

Figure 3. Simulated transverse electron distributions $20 \mathrm{~mm}$ downstream from the split ring for different $\mathrm{THz}$ frequencies and initial electron pulse durations. For a), c), and e), the initial temporal duration was set to zero, while graphs b), d) and f) correspond to $50 \mathrm{fs}$ initial temporal duration. Graphs a) and b), c) and d), and e) and f) correspond to $\mathrm{THz}$ frequencies of $0.3 \mathrm{THz}, 0.6 \mathrm{THz}$, and $0.9 \mathrm{THz}$, respectively. The peak transverse electric field in the gap was $100 \mathrm{MV} / \mathrm{m}$ in all cases.

Figure 4a) shows the behaviour of the streak angle as a function of pulse duration for different $\mathrm{THz}$ frequencies at a value of $100 \mathrm{MV} / \mathrm{m}$. As indicated by the linear fits, the streak angle is linear with pulse duration, yielding a constant angular streak velocity $v_{s, \theta}$ of $10 \mathrm{mrad} / \mathrm{ps}, 23 \mathrm{mrad} / \mathrm{ps}$, and $33 \mathrm{mrad} / \mathrm{ps}$ for frequencies of $0.3 \mathrm{THz}, 0.6 \mathrm{THz}$, and $0.9 \mathrm{THz}$, respectively. The temporal resolution corresponding to these streak velocities depends on the background angular spread of the beam $\sigma_{0}$, which in the case of zero emittance electron beam is dependent on the distortions introduced by the streak field on an electron pulse with zero temporal duration. As seen in Figure 3, such distortions are small but not vanishing. For $100 \mathrm{MV} / \mathrm{m}$, we find $\sigma_{0}=0.026 \mathrm{mrad}, 0.035 \mathrm{mrad}$, and $0.028 \mathrm{mrad}$ for $0.3 \mathrm{THz}, 0.6 \mathrm{THz}$, and $0.9 \mathrm{THz}$, respectively. The temporal resolution is $\Delta t=\sigma_{0} / v_{s, \theta}$, yielding $2.6 \mathrm{fs}, 1.5 \mathrm{fs}$, and $0.9 \mathrm{fs}$ for $0.3 \mathrm{THz}, 0.6 \mathrm{THz}$, and $0.9 \mathrm{THz}$. In Figure $4 \mathrm{~b}$ ), the angular streak velocity is shown versus the split-ring's resonance frequency for different electric fields. The angular streak velocity increases linearly with both frequency and field amplitude, with streak velocity as high as $330 \mathrm{mrad} / \mathrm{ps}$ at $0.9 \mathrm{THz}$ and $1 \mathrm{GV} / \mathrm{m}$. Since the background angular spread varies in the same proportion as the streak velocity, the maximal achievable temporal resolution is still only about $0.9 \mathrm{fs}$ for this case. However, a larger streak field has the advantage of a higher temporal resolution with realistic electron beams that do not have zero emittance. Assuming an angular spread of $\sigma_{0}=0.5 \mathrm{mrad}$ for $0.9 \mathrm{THz}$ and $1 \mathrm{GV} / \mathrm{m}$ yields a temporal resolution of $1.5 \mathrm{fs}$, while the corresponding temporal resolution for the $100 \mathrm{MV} / \mathrm{m}$ case is only $15 \mathrm{fs}$. 

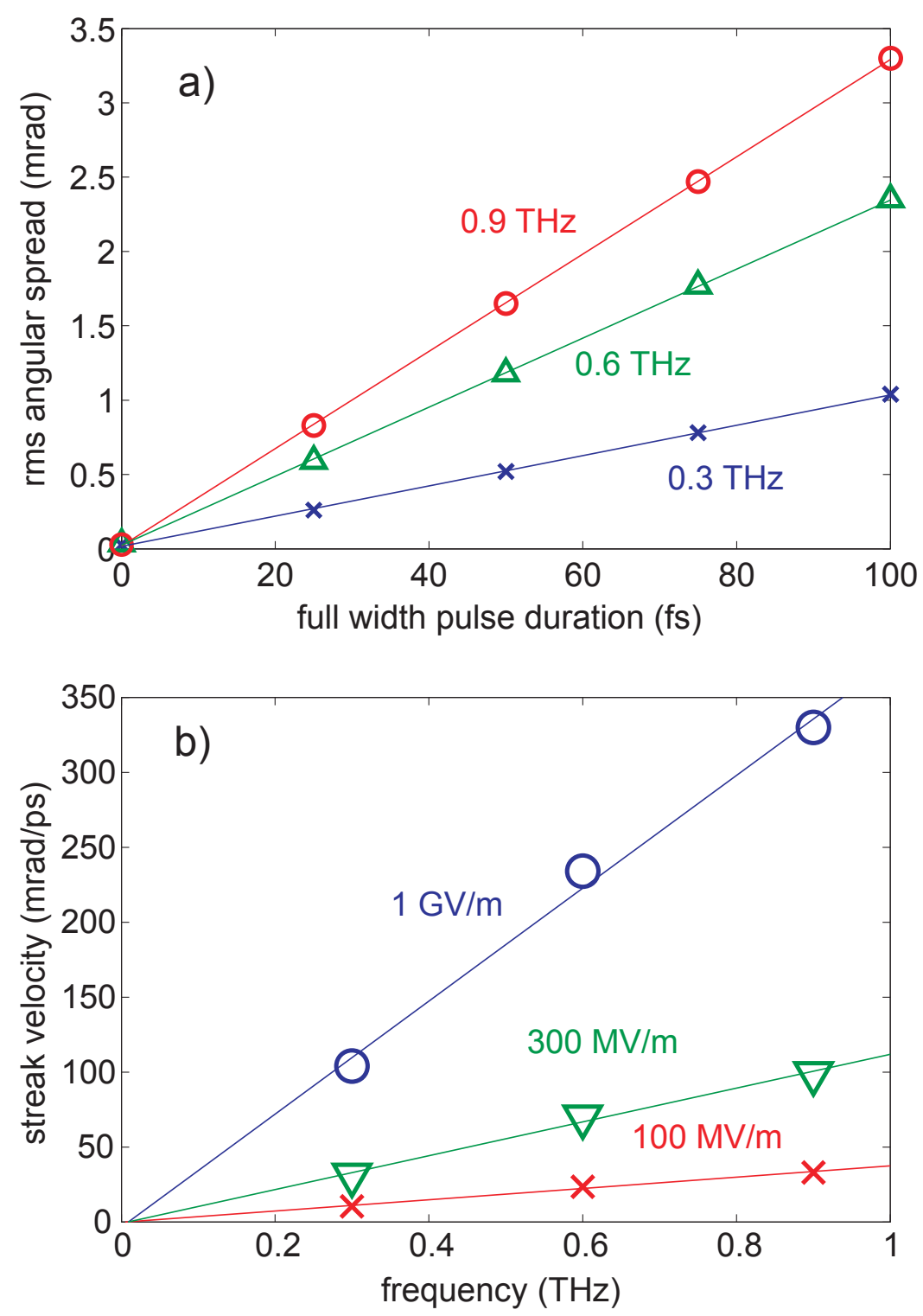

Figure 4. a) Simulated rms angular spread versus temporal pulse duration of $30 \mathrm{kV}$ electron pulses for $0.3 \mathrm{THz}$ (crosses), $0.6 \mathrm{THz}$ (triangles) and $0.9 \mathrm{THz}$ (circles). The linear fits yield angular streak velocities of $v_{s, \theta}=10 \mathrm{mrad} / \mathrm{ps}, 23 \mathrm{mrad} / \mathrm{ps}$ and $33 \mathrm{mrad} / \mathrm{ps}$. The peak electric field was $100 \mathrm{MV} / \mathrm{m}$. b) Angular streak velocity versus resonance frequency for different streak fields. Crosses $100 \mathrm{MV} / \mathrm{m}$, triangles $300 \mathrm{MV} / \mathrm{m}$, and circles $1 \mathrm{GV} / \mathrm{m}$. 
For a more realistic characterization of the streak camera, space charge was included for $4 \mu \mathrm{m} \times 4 \mu \mathrm{m}$ sized electron bunches with a transverse normalized emittance of $\varepsilon_{\mathrm{n}}=1 \mathrm{~nm}$, a bunch charge of $Q=6.4 \times 10^{-9} \mathrm{nC}$ (40 electrons), and a relative rms energy spread of $\Delta E_{\mathrm{r}}=0.1 \%(1 \%)$ in case of $30 \mathrm{keV}(1 \mathrm{MeV})$ electron bunch centre energy. The simulations were performed using the $0.9 \mathrm{THz}$ resonance frequency with a peak transverse electric field of $1 \mathrm{GV} / \mathrm{m}$. For the two cases considered (30 kV and $1 \mathrm{MV}$ electron energy), the split ring to detector distances were $10 \mathrm{~cm}$ and $50 \mathrm{~cm}$, respectively. The results of these simulations are shown in Figure 5. For the $30 \mathrm{keV}$ case, a pulse duration of 3 fs (Figure $5 \mathrm{~b}$ ) already results in significant broadening relative to a $0 \mathrm{fs}$ pulse duration (Figure $5 \mathrm{a}$ ). From the results in Figure $5 \mathrm{c}$, a streak velocity $v_{\mathrm{s}}=33.8 \mu \mathrm{m} / \mathrm{fs}$ at the detector plane can be calculated (corresponding to an angular streak velocity of $v_{s, \theta}$ $=0.338 \mathrm{mrad} / \mathrm{fs}$ ). The corresponding temporal resolution is $\Delta t=\sigma_{0} / v_{s}=1.8 \mathrm{fs}$ with the un-streaked pulse width $\sigma_{0}$ equal to $62 \mu \mathrm{m}$. Similarly for the $1 \mathrm{MeV}$ case, the temporal resolution is $3.7 \mathrm{fs}$. Thus, the proposed streak camera is expected to have a few femtosecond resolution for the measurement of realistic electron bunches in the high energy and in the relativistic energy regimes. It should also be highlighted that the recorded streaking pattern depends negligibly on the chosen relative electron energy bandwidth in the $0-1 \%$ range, making the split ring resonator an ideal tool for electron bunch measurements in UED experiments and femtosecond RF guns such as injectors for FELs or relativistic UED setups.

a) $30 \mathrm{kV}, 0 \mathrm{fs}$
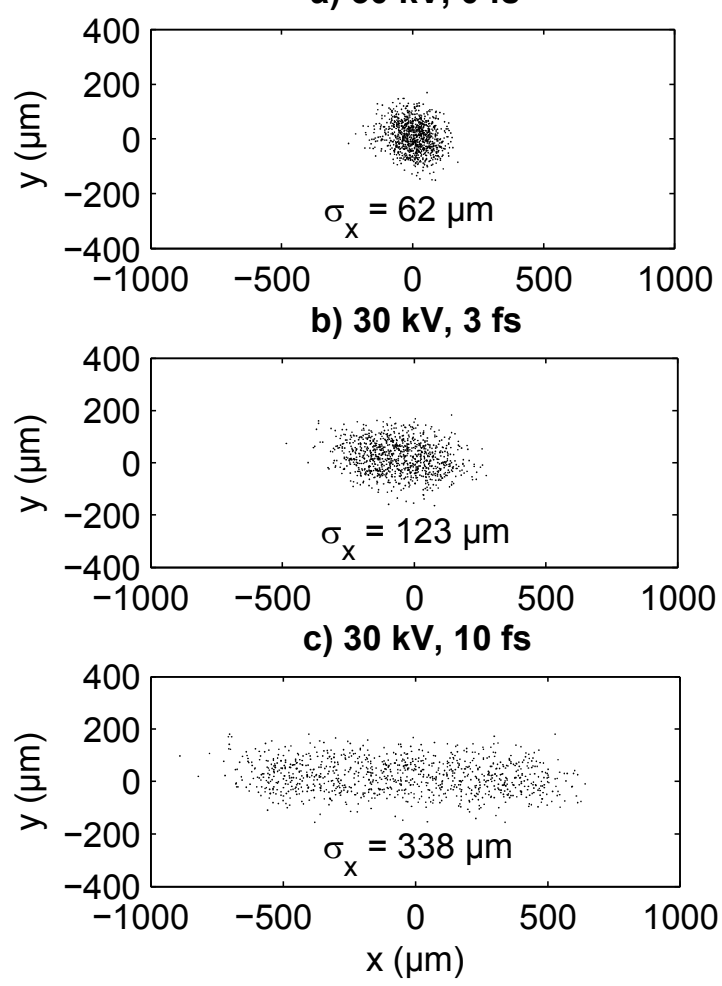

d) $1 \mathrm{MV}, 1 \mathrm{fs}$
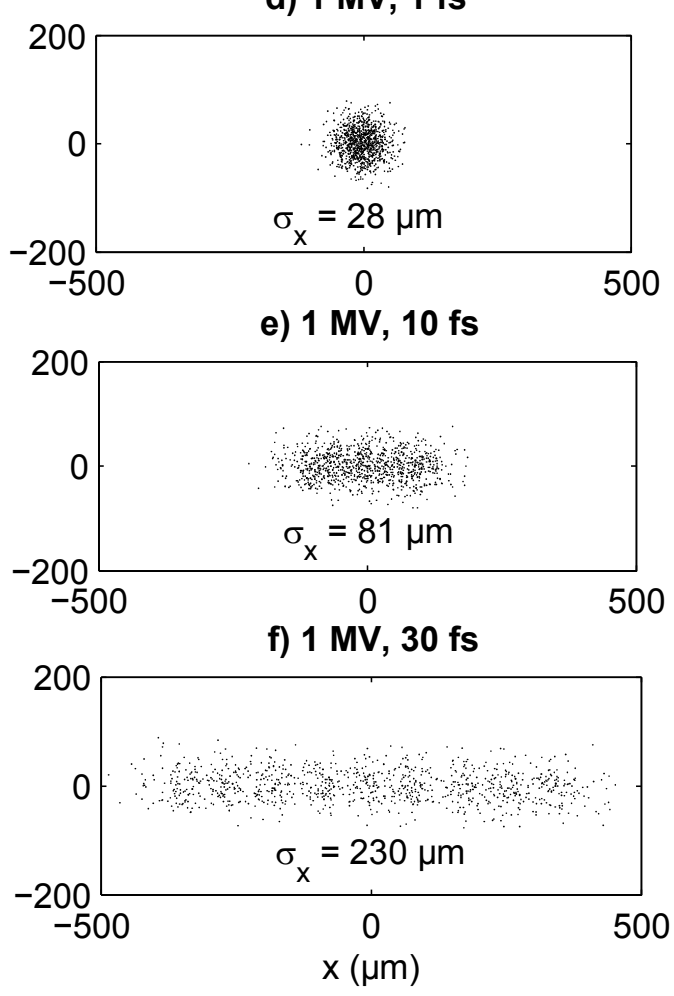

Figure 5. Simulated transverse electron distributions for a $0.9 \mathrm{THz}$ split ring resonator with a peak gap field of $1 \mathrm{GV} / \mathrm{m}$. Electron bunches with an rms size $\sigma_{\mathrm{x}}$ and $\sigma_{\mathrm{y}}=2 \mu \mathrm{m}$, transverse normalized emittance $\varepsilon_{\mathrm{n}}=1 \mathrm{~nm}$ and aerial space charge density $\sigma_{\mathrm{Q}}=10^{7}$ electrons $/ \mathrm{mm}^{2}$ were propagated through the split ring resonator towards the detector which was located $10 \mathrm{~cm}$ and $50 \mathrm{~cm}$ further along the beam line for the $30 \mathrm{keV}$ (sub-figures a) through c)) and $1 \mathrm{MeV}$ (sub-figures d) through f)) respectively. Space charge effects are taken into account in this simulation, and the rms bunch size in the x- direction $\sigma_{\mathrm{x}}$ are indicated in the figure. The relative rms energy spread was assumed to be $0.1 \%$ and $1 \%$ for the $30 \mathrm{keV}$ and $1 \mathrm{MeV}$ cases, respectively. 


\section{DISCUSSION}

The proposed THz-driven streak camera is promising for temporal electron pulse measurements in the few femtosecond up to few hundred femtosecond time domain. While the temporal resolution depends on a variety of parameters, the total streaking window is determined by the resonance frequency of the split-ring resonator alone. In addition, we find no discernable effect of the energy bandwidth of the measured electron bunches on the streak pattern up to relative bandwidths of $1 \%$, thereby making our THz streak camera an ideal tool for electron bunch diagnostics in beam lines with highly charged bunches and compressor elements, as found in UED setups, synchrotrons, and XFEL's.

\section{REFERENCES}

[1] Miller, R. J. D.. "Mapping Atomic Motions with Ultrabright Electrons: The Chemists' Gedanken Experiment Enters the Lab Frame", Phys. Chem. 65, 583 (2014).

[2] Miller, R. J. D. "Femtosecond Crystallography with Ultrabright Electrons and X-rays: Capturing Chemistry in Action", Science 343, 1108 (2014).

[3] Ihee, $H$. et al. "Direct imaging of transient molecular structures with ultrafast diffraction", Science 291, 458 (2001).

[4] Siwick, B. J., Miller, R. J. D. "An atomic-level view of melting using femtosecond electron diffraction". Science 302, 1382 (2003).

[5] Siwick, B.J., Dwyer., J.R., Jordan, R.E., Miller R. J. D., "Femtosecond electron diffraction studies of strongly driven structural phase transitions", Chem. Phys. 299, 285 (2004).

[6] Eichberger, M. et al. "Snapshots of cooperative atomic motions in the optical suppression of charge density waves". Nature 468, 799-802 (2010).

[7] Erasmus, N. et al. "Ultrafast dynamics of charge density waves in $4 \mathrm{H}(\mathrm{b})-\mathrm{TaSe} 2$ probed by femtosecond electron diffraction”. Phys. Rev. Lett. 109, 167402 (2012).

[8] Gao, M. et al. "Mapping molecular motions leading to charge delocalization with ultrabright electrons". Nature 496, 343-346 (2013).

[9] Hebeisen, C., T. et al. "Femtosecond electron pulse characterization using laser ponderomotive scattering". Opt. Lett. 31, 3517-3519 (2006).

[10] Wang, X. et al. "Measurement of femtosecond electron pulse length and the temporal broadening due to space charge". Rev. Sci. Instrum. 80, 13902 (2009).

[11] Hebeisen, C. T. et al. "Grating enhanced ponderomotive scattering for visualization and full characterization of femtosecond electron pulses". Opt. Express 16, 3335 (2008).

[12] Kassier, G. H. et al., "A compact streak camera for $150 \mathrm{fs}$ time resolved measurement of bright pulses in ultrafast electron diffraction". Rev. Sci. Instrum. 81, 105103 (2010).

[13] Krüger, M. et al. "Interaction of ultrashort laser pulses with metal nanotips: a model system for strong-field phenomena". New J. Phys. 14, 085019 (2012).

[14] Mustonen, A., Beaud, P., Kirk, E., Feurer, T. \& Tsujino, S., "Efficient light coupling for optically excited highdensity metallic nanotip arrays". Nature Sci. Rep. 2, 915 (2012).

[15] Hoffrogge, J. et al. "A tip-based source of femtosecond electron pulses at 30keV". J Appl. Phys. 115, 094506 (2014).

[16] Krüger, M., Schenk, M., Förster, M. \& Hommelhoff, P. "Attosecond physics in photoemission from a metal nanotip". J. Phys. B: At. Mol. Opt. Phys. 45, 074006 (2012).

[17] Wachter, G. et al. "Electron rescattering at metal nanotips induced by ultrashort laser pulses". Phys. Rev. B 86, $035402(2012)$. 
[18] van Oudheusden, T. et al., "Compression of subrelativistic space-charge-dominated electron bunches for singleshot femtosecond electron diffraction". Phys. Rev. Lett. 105, 264801 (2010).

[19] Veisz, L. et al. "Hybrid dc-ac electron gun for fs-electron pulse generation". New J. Phys. 9, 451 (2007)

[20] Kassier, G.H. et al. "Photo-triggered pulsed cavity compressor for bright electron bunches in ultrafast electron diffraction”, Appl. Phys. B 109, 249 (2012).

[21] Fabiańska, J., Kassier, G., Feurer, T., "Split ring resonator based THz-driven electron streak camera featuring femtosecond resolution", Nature Scientific Reports, vol.: 4, pp.: 5645, (2014).

[22] Bagiante, S., Enderli, F., Fabiańska, J., Sigg, H. \& Feurer, T. "Giant Electric Field Enhancement in Split Ring Resonators Featuring Nanometer-Sized Gaps", Nature Scientific Reports, vol 5, pp.: 8051 (2015).

[23] Ruchert, C., Vicario, C. \& Hauri, C. P. "Scaling Sub-mm single-cycle transients towards MV/cm fields via optical rectification in organic crystal OH1". Opt. Lett. 37, 899 (2012).

[24] Sell, A., Leitenstorfer, A. \& Huber, R.. "Phase-locked generation and field-resolved detection of widely tuneable terahertz pulses with amplitudes exceeding $100 \mathrm{MV} / \mathrm{cm}$ ". Opt. Lett. 33, 2767 (2008).

[25] Merbold, H., Brunner, F., Cannizzo, A., Feurer, T. "Near Field Enhancement for THz Switching and THz Nonlinear Spectroscopy Applications", Chimia 65, 316 (2011). 